\title{
Synaptic Plasticity (and the Lack Thereof) in Hippocampal CA2 Neurons
}

\author{
Meilan Zhao, ${ }^{1}$ Yun-Sik Choi, ${ }^{2}$ Karl Obrietan, ${ }^{2}$ and Serena M. Dudek ${ }^{1}$ \\ ${ }^{1}$ National Institute of Environmental Health Sciences, National Institutes of Health, Research Triangle Park, North Carolina 27709 , and ${ }^{2} \mathrm{Ohio}$ State \\ University, Department of Neuroscience, Columbus, Ohio 43210
}

\begin{abstract}
The hippocampus is critical for some forms of memory and spatial navigation, but previous research has mostly neglected the CA2, a unique region situated between CA3 and CA1. Here, we show that CA2 pyramidal neurons have distinctive physiological characteristics that include an unprecedented synaptic stability. Although basal synaptic currents in CA1 and CA2 are quite similar, synaptic plasticity including long-term potentiation and long-term depression is absent or less likely to be induced with conventional methods of stimulation in CA2. We also find that CA2 neurons have larger leak currents and more negative resting membrane potentials than CA1 neurons, and consequently, more current is required for action potential generation in CA2 neurons. These data suggest that the molecular "conspiracy against plasticity" in CA2 makes it functionally distinct from the other hippocampal CA regions. This work provides critical insight into hippocampal function and may lead to an understanding of the resistance of CA2 to damage from disease, trauma, and hypoxia.
\end{abstract}

Key words: long-term potentiation; long-term depression; TREK-1 channels; STEP; ERK; CREB

\section{Introduction}

Is CA2 a distinct subfield of the hippocampus, or is it merely a transition region between $\mathrm{CA} 1$ and $\mathrm{CA} 3$ ? Evidence for the former is that CA2 is spared in several models of hypoxia/ischemia (Kirino, 1982; Sadowski et al., 1999) (but see Han et al., 2005), and it possesses a unique pathology in schizophrenic patients (Benes et al., 1998; Benes and Todtenkopf, 1999). Moreover, the CA2 neurons of normal aged humans without Alzheimer's disease often contain large, lipofuscin-containing deposits not seen in other CA areas (Braak, 1980), suggestive of distinct protein or lipid metabolism in this area. Thus, CA2 of hippocampus appears to be dissimilar from the other CA regions with respect to death and survival from injury or age. Anatomically, CA2 pyramidal neurons closely resemble CA3 neurons in size and dendritic branching patterns (Lorente de No, 1934; Woodhams et al., 1993; Ishizuka et al., 1995), but share with CA1 features that include the lack of large, thorny spines characteristic of the mossy-fiber synapses from the dentate gyrus. Molecularly, CA2 can be clearly delineated from its neighboring CA regions by expression patterns of some genes (Zhao et al., 2001; Lein et al., 2004, 2005). Also, some extracellular matrix components and oligodendro-

\footnotetext{
Received April 12, 2007; accepted Sept. 14, 2007.

This work was supported by the Intramural Research Program of the National Institutes of Health, by the National Institute of Environmental Health Sciences (S.M.D.), and by National Institute of Neurological Disorders and Stroke Grant NS47176 (K.O.). We thank Drs. David Armstrong, Marc Sommer, and Bin Tu for their helpful advice and Mr. Gregory Gardner for some data analysis.

Correspondence should be addressed to Serena M. Dudek, National Institute of Environmental Health Sciences, National Institutes of Health, P.0. Box 12233, MD F2-04, Research Triangle Park, NC 27709. E-mail: dudek@niehs.nih.gov.

D0I:10.1523/JNEUROSCI.4094-07.2007

Copyright $\odot 2007$ Society for Neuroscience $\quad$ 0270-6474/07/2712025-08\$15.00/0
}

cytes are found in greater abundance there than in CA1 and CA3 (Berger and Frotscher, 1994; Bruckner et al., 2003).

In view of these differences in molecular makeup and resistance to injury, we tested the hypothesis that CA2 neurons in rat hippocampus are similarly resistant to synaptic plasticity when compared with CA1 neurons. Whole-cell voltage- and currentclamp recordings were used to examine resting membrane potential, leak currents, and action potential and synaptic properties. We found that several of these properties distinguish CA2 from CA1. Most striking, however, was the apparent stability of synaptic responses in CA2 that included a resistance to long-term potentiation (LTP) and long-term depression (LTD) induction protocols relative to the other CA subfields.

\section{Materials and Methods}

Electrophysiological recordings. Hippocampal slices were prepared from Sprague Dawley rats (generally 11-21 d of age) or from C57BL/6J mice (also 11-21 d of age). Under deep anesthesia, animals were decapitated, and the brains were rapidly removed. Coronal brain slices ( $350 \mu \mathrm{m}$ thick) containing the hippocampus were cut using a vibrating blade microtome in ice-cold sucrose-substituted artificial CSF (ACSF) containing the following (in mM): 240 sucrose, $2.0 \mathrm{KCl}, 1 \mathrm{MgCl}_{2}, 2 \mathrm{MgSO}_{4}, 1 \mathrm{CaCl}_{2}, 1.25$ $\mathrm{NaH}_{2} \mathrm{PO}_{4}, 26 \mathrm{NaHCO}_{3}$, and 10 glucose, which was bubbled continuously with $95 \% \mathrm{O}_{2} / 5 \% \mathrm{CO}_{2}$ to obtain a $\mathrm{pH}$ of 7.4 . Freshly cut slices were placed in an incubating chamber with ACSF and recovered for at least $1 \mathrm{~h}$. For electrophysiological recordings, slices were transferred to a recording chamber in which they were bathed continuously with ACSF. Recordings were made from CA2 neurons only when the CA2 could be visually distinguished from CA1; CA2 has the larger neurons (supplemental Fig. 1, available at www.jneurosci.org as supplemental material). The average ages of the animals were similar in CA1 and CA2 recordings (13.00 \pm 0.35 and $12.97 \pm 0.29 \mathrm{~d}$, respectively), as generally, recordings were alternated between the two areas. In some cases, not included in these averages, animals $>4$ weeks of age were used for supplemental data. 
Although most of the data presented were from recordings performed at room temperature, similar results were obtained when experiments were performed at $34.5^{\circ} \mathrm{C}$ (supplemental material, available at www.jneurosci.org).

In most cases, whole-cell recording of CA1 neurons were made with patch pipettes (3-5 M $\Omega$ ) filled with solution containing the following (in mM): $120 \mathrm{~K}$-gluconate, $10 \mathrm{KCl}, 3 \mathrm{MgCl}_{2}, 0.5 \mathrm{EGTA}, 40 \mathrm{HEPES}, 2 \mathrm{Na}_{2}$ ATP, $0.3 \mathrm{Na}-\mathrm{GTP}$, with $\mathrm{pH}$ adjusted to 7.2 by $\mathrm{NaOH}$. For the pairinginduced LTP experiments, the internal solution contained the following (in mM): 115 Cs-methanesulfonate, $20 \mathrm{CsCl}, 2.5 \mathrm{MgCl}_{2}, 0.6$ EGTA, 10 HEPES, $4 \mathrm{Na}_{2}$-ATP, $0.4 \mathrm{Na}$-GTP, and 10 phosphocreatine disodium salt. For the measurements of NMDA receptor (NMDAR) currents, the internal solution contained the following (in $\mathrm{mm}$ ): $102 \mathrm{CsOH}, 102$ D-gluconate, $3.7 \mathrm{NaCl}$, 10 BAPTA, 0.2 EGTA, 20 HEPES, 4 Mg-ATP, 0.3 Na-GTP, 5 lidocaine $N$-ethyl bromide (QX314), and 5 tetraethylammonium chloride. Perforated patch recordings were made using $100 \mathrm{mg} / \mathrm{ml}$ nystatin added to the pairing-LTP solution. For the leak current and NMDAR measurements, $5 \mathrm{~mm}$ QX314 was included in the standard internal solution for blockade of sodium-dependent action potentials.

Standard ACSF consisted of the following (in mM): $124 \mathrm{NaCl}, 2.5 \mathrm{KCl}$, $2 \mathrm{MgCl}_{2}, 2 \mathrm{CaCl}_{2}, 1.25 \mathrm{NaH}_{2} \mathrm{PO}_{4}, 26 \mathrm{NaHCO}_{3}$, and 17 D-glucose. For leak current measurements, the external solution contained $3 \mathrm{~mm} \mathrm{KCl,} 1$ $\mathrm{mm}$ tetrodotoxin, and $50 \mathrm{~mm}$ ZD7288, and D-gluconic acid sodium salt (140 mM) was substituted for the sodium chloride. Reversal potentials could be shifted by changing the external potassium concentration. For NMDAR currents, standard ACSF was used with the following modifications (in $\mathrm{mm}$ ): $3 \mathrm{KCl}, 4 \mathrm{CaCl}_{2}$, and $4 \mathrm{MgCl}_{2}, 1$ glycine, 50 picrotoxin, and 20 CNQX. Miniature EPSCs (mEPSCs) were recorded in standard ACSF with $0.1 \mathrm{~mm} \mathrm{MgSO}_{4}$ and $0.5 \mathrm{~mm}$ picrotoxin before and after application of $50 \mathrm{~mm}$ APV. Membrane potential measurements and action potential thresholds were corrected for the recorded junction potential $(10 \mathrm{mV})$. Stimulating electrodes (cluster-type) were obtained from FHC (Bowdoinham, ME). LTP was induced with either two $1 \mathrm{~s}$ bursts of stimulation delivered at $100 \mathrm{~Hz}$ in current-clamp mode, repeated once with a $30 \mathrm{~s}$ interval, or with a pairing protocol $[1.5 \mathrm{~min}$ of $3 \mathrm{~Hz}$ stimulation (270 pulses) paired with depolarization to $0 \mathrm{mV}$ in voltage-clamp mode]. LTD was induced with $7.5 \mathrm{~min}$ of stimulation at $2 \mathrm{~Hz}$, with cells held at $-75 \mathrm{mV}$ in voltage clamp. For LTP in the perforated-patch recordings, the baseline synaptic responses were allowed to stabilize after the access resistance fell below $400 \mathrm{~m} \Omega$ (generally 10-15 min). Otherwise, the baselines for LTP experiments in whole-cell mode were always 5 min (for both CA1 and CA2). Uncompensated series resistances $(<20$ $\mathrm{m} \Omega$ ) did not differ significantly between CA1 and CA2 (CA1, $12.71 \pm$ 0.15 ; CA2, $13.4 \pm 0.78$ ).

Miniature EPSCs were recorded at $-60 \mathrm{mV}$ over two 3 min periods, both before, and after application of $50 \mathrm{~mm}$ D-APV. NMDAR components were calculated by subtracting the average of traces acquired during APV from the averaged traces before the APV.

For determination of action potential thresholds and spike accommodation, whole-cell recordings were made in current-clamp mode. Current pulses of $180 \mathrm{~ms}$ duration in $0.2 \mathrm{nA}$ increments were applied to CA1 and CA2 pyramidal cells, and the membrane potentials at which point the cells fired action potentials determined by using the method outlined by Henze et al. (2000). Briefly, the action potential threshold was determined by finding the voltage at the point at which the third derivative crossed from negative to positive before the peak of the first derivative. Accommodation was expressed as a ratio of the interval between the first two action potentials.

Seizures and staining. Status epilepticus was induced in male C57BL/6 male mice (7-8 weeks of age) via the intraperitoneal injection of pilocarpine. Animals were initially injected (intraperitoneally) with $1 \mathrm{mg} / \mathrm{kg}$ atropine methyl nitrate $30 \mathrm{~min}$ before being injected with pilocarpine $(325 \mathrm{mg} / \mathrm{kg}$, diluted in physiological saline; Sigma, St. Louis, MO). Status epilepticus was defined as a continuous motor seizure of stage 4 (rearing and falling), 5 (loss of balance, continuous rearing and falling), or stage 6 (severe tonic-clonic seizures) (Racine, 1972). After $15 \mathrm{~min}$ of status epilepticus, mice were transcardially perfused with cold saline, followed by $4 \%$ paraformaldehyde in PBS (10 mm; $\mathrm{pH} 7.4)$ under ketamine/xylazine anesthesia. Brains were postfixed in $4 \%$ paraformaldehyde for $4 \mathrm{~h}$ at $4^{\circ} \mathrm{C}$ and cryoprotected with $30 \%$ sucrose in PBS. Coronal sections (40 $\mu \mathrm{m}$ ) through the dorsal hippocampus were prepared using a freezing microtome.

For immunohistochemistry, brain sections were washed with PBS and incubated in $0.3 \%$ hydrogen peroxide/PBS for $20 \mathrm{~min}$ to eliminate endogenous peroxidase activity. After several washes with PBS, sections were blocked with $10 \%$ normal goat serum in PBS, followed by overnight incubation with rabbit anti-phosphorylated extracellular signalregulated kinase (pERK) antibody (Cell Signaling Technology, Beverly, MA; 1:2000) or mouse monoclonal anti-striatal enriched tyrosine phosphatase (STEP) antibody (Novus Biologicals, Littleton, CO; 1:2000) at $4^{\circ} \mathrm{C}$. Sections were then processed using the $\mathrm{ABC}$ staining method (Vector Laboratories, Burlingame, CA). Nickel-intensified DAB (Vector Laboratories) was used to visualize the signal. Photomicrographs were captured using a 16 bit digital camera (Micromax YHS 1300; Princeton Instruments, Trenton, NJ) mounted on a Leica (Nussloch, Germany) DM IRB microscope. A similar protocol was used for staining of TREK1 (Alomone Labs, Jerusalem, Israel; 1:500) and calbindin D28K (Chemicon; 1:500), except that secondary antibodies were conjugated with Alexa Fluor (Invitrogen, Carlsbad, CA; 1:100). For identification of a limited number of CA2 neurons, $200 \mathrm{~mm}$ Oregon Green or $0.4 \%$ biocytin conjugated with Alexa Fluor 488 was added to the internal electrode solution, and after recording, the slices were fixed overnight.

\section{Results}

\section{LTP expression is impaired in hippocampal CA2 neurons}

The hippocampus, more than any other brain region, is distinguished for its plasticity of synaptic responses. High-frequency synaptic stimulation typically results in a long-lasting increase in the size of the synaptic response (LTP) in the dentate gyrus, CA3, and CA1 of hippocampus (Bliss and Lomo, 1973; Alger and Teyler, 1976), but never has LTP been assessed in CA2. Using wholecell recordings, we tested whether $100 \mathrm{~Hz}$ synaptic stimulation could induce LTP in CA2 neurons with the postsynaptic neuron in current-clamp mode. In most CA2 neurons, no potentiation of the synaptic currents was observed, and in some cases, a depression was induced (supplemental Fig. 2, available at www. jneurosci.org as supplemental material). This is in contrast to CA1, in which LTP can be readily induced in most neurons. To rule out the possibility that synapses onto CA2 neurons do not express LTP because they cannot follow high frequencies of stimulation, and to insure that the postsynaptic cell was adequately depolarized, we tested whether a pairing protocol $(3 \mathrm{~Hz}$ stimulation paired with depolarization to $0 \mathrm{mV}$ ) could induce LTP in CA2 neurons. As with the $100 \mathrm{~Hz}$ stimulation, we failed to induce LTP in CA2 neurons using this pairing protocol (Fig. 1), both with and without picrotoxin in the bath to block $\mathrm{GABA}_{\mathrm{A}}$ inhibitory currents or when a perforated patch configuration was used (supplemental Fig. 3, available at www.jneurosci.org as supplemental material). We consistently induced LTP when using these protocols in CA1. The pairing protocol was also ineffective at inducing LTP even when the recordings were made closer to physiological temperature or from slices prepared from older rats (supplemental Fig. 3, available at www.jneurosci.org as supplemental material). Neither stimulation at higher frequencies, such as those most effective for inducing LTP in dentate gyrus $(200 \mathrm{~Hz}$; eight pulses; repeated eight times), nor inclusion of a phosphatase inhibitor (1 mM okadaic acid) to block LTD, was effective at aiding in LTP induction in CA2 ( $n=4$ and 3$)$ (data not shown).

To rule out that this lack of LTP in CA2 was unique to the rat, we tested the pairing protocol for its ability to induce LTP in mouse hippocampus. Although a potentiation was induced immediately after the pairing that was indistinguishable from that in mouse CA1 (supplemental Fig. 3, available at www.jneurosci.org as supplemental material), the potentiation rapidly decayed to 


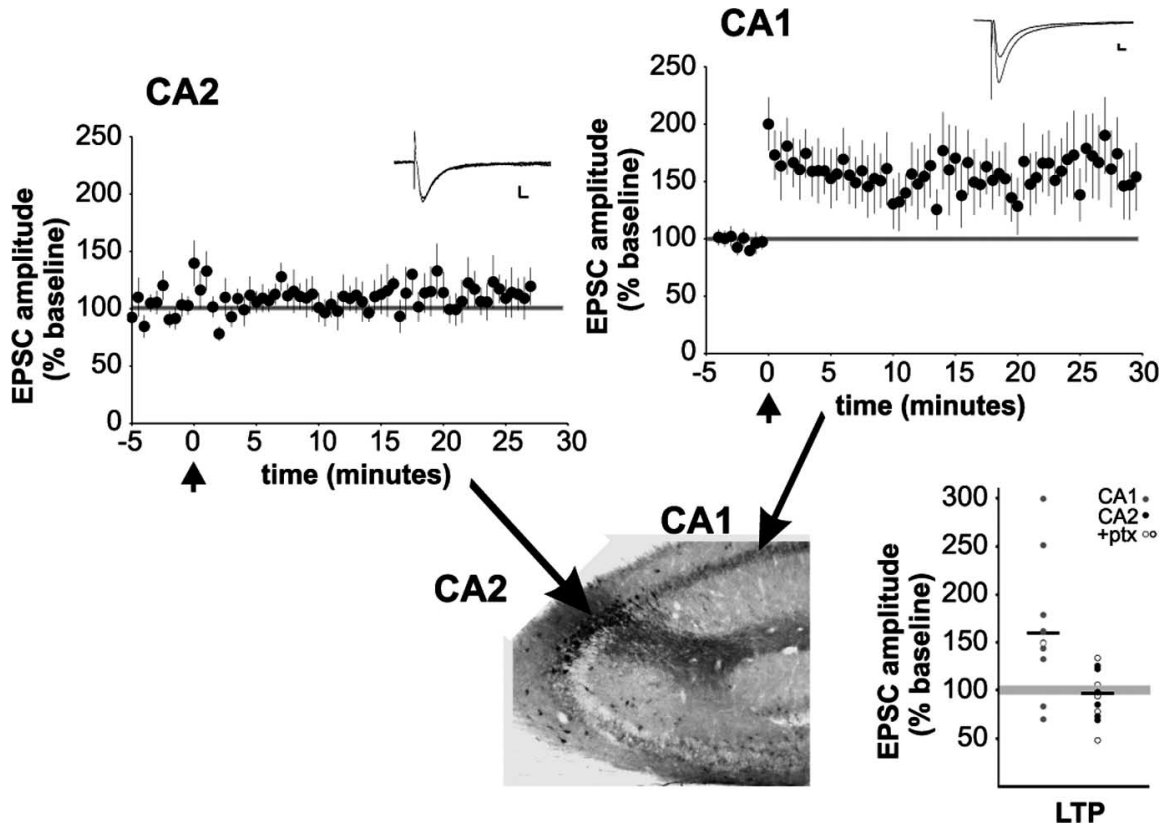

Figure 1. Long-term potentiation is absent from CA2 neurons. Shown compared with LTP induced in rat CA1 neurons, a pairing protocol ( $3 \mathrm{~Hz}$, with depolarization to $0 \mathrm{mV}$ ) is ineffective at inducting LTP in rat CA2 neurons. LTP is similarly impaired in the mouse CA2, relative to CA1, but shows greater short-term, or posttetanic potentiation than that seen in the rat (supplemental Fig. 3, available at www.jneurosci.org as supplemental material). The insets are traces from representative experiments from each area. Calibration: 25 pA, $10 \mathrm{~ms}$. Amount of LTP induced from individual cases is shown, expressed as a percentage of the baseline value: CA1 (gray) and CA2 (black). The open circles represent those experiments performed in $50 \mu \mathrm{m}$ picrotoxin. Hippocampal CA2 is illustrated with staining for STEP, which has been described previously (Boulanger et al., 1995). Error bars indicate SEM.
Patterns of expression of NMDA receptor mRNA and agonist binding show that the major NMDA subunits are expressed evenly across the hippocampus, or with CA2 being similar to CA3 (Buller et al., 1994; Wenzel et al., 1995). To rule out that NMDA receptors are rendered less functional in CA2 neurons, perhaps by STEP, which is enriched in CA2 (Boulanger et al., 1995) and has been demonstrated to negatively regulate NMDARs (Pelkey et al., 2002), we recorded synaptic NMDA receptor responses in the presence of an AMPA-type glutamate receptor antagonist $(\mathrm{CNQX})$ and $\mathrm{a} \mathrm{GABA}_{\mathrm{A}}$ receptor antagonist (bicuculline). We found that NMDA receptor-mediated currents were indistinguishable between CA1 and CA2 at a range of stimulus intensities (Fig. 2D). Furthermore, the NMDAR-dependent components of mEPSCs and their ratio to AMPAR-dependent components of the mEPSCs do not differ between CA1 and CA2 (Fig. 2C), indicating that impairment of NMDA receptor function cannot explain the reduced plasticity of CA2. Analyses of NMDA receptor-dependent waveforms to distinguish between contributions of NR2A and NR2B NMDA receptor subunits, which are differentially implicated in LTP and LTD, respectively (Liu the baseline level within $10 \mathrm{~min}$, suggesting that critical components of the LTP consolidating pathway are missing in the CA2 of both rats and mice. Although we cannot rule out the possibility that some other stimulation may prove successful in inducing LTP, these data do demonstrate that LTP, if present in CA2, is fundamentally different from that in CA1 (and CA3) in that it is only rarely induced with current standard protocols from a normal baseline state.

\section{Synaptic responses do not differ between CA1 and CA2}

With LTP so severely impaired in CA2 relative to CA1 and CA3, we sought to determine whether synaptic properties could explain the deficit, although the size of the baseline synaptic responses used in the plasticity experiments did not differ from one another significantly $(87.61 \pm 7.11 \mathrm{pA}$ in CA1; $81.08 \pm 6.31$ in CA2; $n=33$ and 31, respectively; includes data from LTD experiments below). To determine whether synaptic responses were less effective or otherwise different in CA2, an input-output curve was constructed (Fig. 2A). We found that synaptic responses evoked over a range of stimulation intensities were not significantly different between CA2 and CA1, consistent with the fact that both areas receive much of the same type of synaptic inputs from the CA3 (Lorente de No, 1934). mEPSC amplitudes also did not differ significantly between CA1 and CA2 (CA1, $10.02 \pm 1.31 \mathrm{pA}$; CA2, $8.56 \pm 1.48 \mathrm{pA} ; n=9$ each; $p>0.4)$. Presynaptic function, as assessed by mEPSC frequency and paired-pulse facilitation (PPF), was similar in CA1 and CA2 (Fig. $2 B, C)$, but PPF was slightly larger in CA2 at the smallest interval tested $(p<0.03)$. These data do not explain the lack of LTP in $\mathrm{CA} 2$, because greater paired-pulse facilitation has been associated with lower probability of release and possibly more LTP (Schulz et al., 1994) (but see Asztely et al., 1996). et al., 2004), also revealed no difference between CA1 and CA2 neurons (data not shown). The voltage dependences of NMDARdependent responses similarly did not differ between the two areas (data not shown). Thus, a reduced presence/function of NMDARs cannot explain the relative lack of LTP of CA2.

\section{LTD is heterogeneously expressed in hippocampal \\ CA2 neurons}

Do the properties that limit LTP in CA2 similarly inhibit LTD, considering that both LTD and LTP rely on NMDA receptor activation and postsynaptic calcium influx (Malenka and Bear, 2004)? To answer this question, we tested a stimulation protocol typically very effective in CA1 for its ability to induce LTD in CA2 ( $2 \mathrm{~Hz}$ at $-75 \mathrm{mV}$ holding potential in whole-cell voltage clamp) (Fig. $3 A, B$ ). In contrast to LTP, LTD could be induced in some CA2 neurons that was of equal magnitude to that induced in sponse to the low-frequency stimulation; in more than one-third of CA2 neurons, LTD could not be induced [LTD in 21 of 34 cells $(61.8 \%)$ in CA2, vs 20 of 23 cells $(86.9 \%)$ in CA1 (Fisher's exact test, $p=0.039$; unequal variance $t$ test, $p<0.05$ ) ] (Fig. $3 B$ ). The initial size of EPSCs in "depressing" CA2 cells (87.5 pA) was not significantly different from the initial size of the "nondepressing" CA2 cells ( $68.7 \mathrm{pA} ; p>0.24)$, but because the initial EPSC size is set by the experimenter at the beginning of each LTD experiment, and thus potentially normalizing different-sized responses, we examined in a post hoc analysis whether the cells in which LTD was induced (LTD cells) had a different sensitivity to electrical stimulation than those in which LTD could not be induced (noLTD cells). We found that they did not differ (Fig. 3C). These findings suggest that whatever is responsible for limiting LTP in CA2, it is not impacting LTD in all CA2 neurons, and it cannot be CA1. Unlike CA1, however, CA2 showed a heterogeneous re- 
A

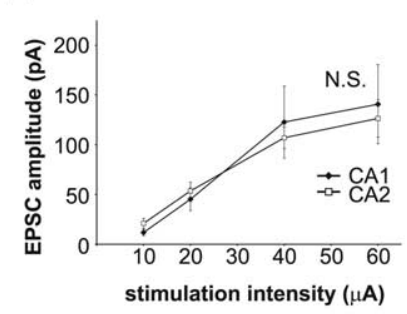

C

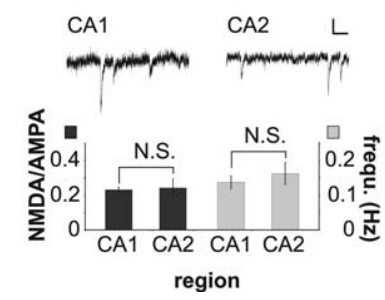

B

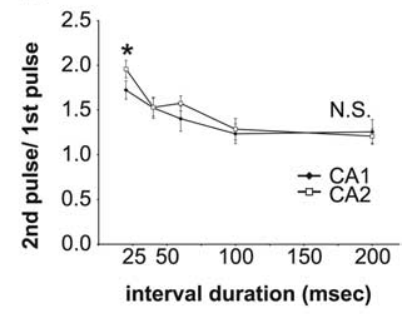

D

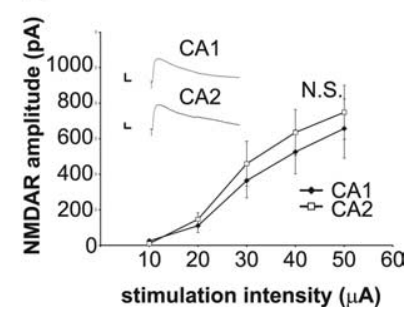

Figure 2. Synaptic properties recorded in CA1 and CA2 neurons. Excitatory synaptic currents in response to electrical stimulation were not significantly different between CA2 and CA1 neurons. $A$, A range of stimulation intensities up to $60 \mathrm{~mA}$ stimulation were used to evoke synaptic responses recorded in CA1 (black diamonds) and CA2 neurons (white squares). At no intensity were the two significantly different ( $n=8$ cells each). $\boldsymbol{B}$, The level of PPF was determined by delivering two electrical pulses at varying intervals. The ratio of the amplitude of the second response to the amplitude of the first response is plotted according to the interval between the two pulses. PPF in CA2 was very similar to PPF in CA1, but was larger at the smallest interval ( ${ }^{*} p<0.03 ; n=10$ cells each). $C$, mEPSC $s$ did not differ between CA1 and CA2. Shown are example traces, NMDA/AMPA ratios, and frequency of events ( $n=9$ neurons each). Calibration: 10 pA, $100 \mathrm{~ms}$. D, Representative NMDA receptor currents from CA1 and CA2 neurons, recorded at $+40 \mathrm{mV}$. Calibration: $20 \mathrm{pA}$ (CA1) and $10 \mathrm{pA}$ (CA2), $25 \mathrm{~ms}$. NMDA receptordependent current amplitudes did not differ significantly between CA1 and CA2 at several stimulation intensities ( $n=10$ in each case; $p>0.2$ at all stimulation intensities, $t$ test). Error bars indicate SEM.

explained by initial "plasticity state" of the synapses. These findings also demonstrate that LTD induction is heterogeneously expressed in CA2, much like the expression of some molecules such as chromogranin A and the vasopressin $1 \mathrm{~b}$ receptor (Munoz, 1990; Woodhams et al., 1993; Young et al., 2006).

\section{CA1 and CA2 membrane properties differ}

To investigate whether CA1 and CA2 neurons could be otherwise discerned electrophysiologically, and because TREK-1 channels are concentrated in rat CA2 (Talley et al., 2001), we sought to determine whether leak-like currents were different between the two areas. Using whole-cell voltage clamp, we found that currents in response to voltage steps were larger in CA2 neurons than in CA1 (Fig. $4 A, B)$ ( $n=10$ each). Currents measured from CA3 neurons were intermediate between the two (data not shown). Neurons possessing greater number of these background (leak) currents should have a greater (more negative) membrane potential than others containing fewer channels, assuming that they contribute the largest proportion to the membrane potential (Goldman, 1943). Consistent with this, we found that, indeed, CA2 had a highly significant difference in membrane potential from CA1, and it was more negative (Fig. $4 C)(\mathrm{CA1}, 69.36 \pm 0.59$; CA2, $73.57 \pm 0.40 ; p<0.001 ; n=20$ cells each). Because other two-pore domain family members of potassium channels, including TASK-1, TASK-3, TWIK-1, are all represented in CA2 and CA1 differently, with TASK-3 enriched in CA1 (Talley et al., 2001), we cannot rule out that the currents are attributable to

some channel other than TREK-1, or a signaling molecule differentially expressed in CA2.

If CA2 cells have leakier membranes, it follows that more current will be required for action potential generation. To establish whether CA2 neurons are more difficult to fire relative to CA1, we determined the minimum stimulus for action potential generation, and the action potential thresholds. In current-clamp recording mode, current pulses of $180 \mathrm{~ms}$ duration in $0.2 \mathrm{nA}$ increments were applied to CA1 and CA2 pyramidal cells, and the membrane potential at which point the cells fired action potentials was determined (Fig. 5A). We found that more current, on average, was required to generate action potentials in CA2 neurons than CA1 neurons, consistent with our finding of bigger leak currents in CA2 neurons (Fig. 5B). This increased current requirement for action potential generation is not entirely explained by the more negative resting membrane potential in CA2 neurons, because the threshold membrane potential itself was more depolarized (less negative) in CA2 neurons (Fig. 5B). These data do not, however, explain the lack of LTP induction in CA2, because the number of action potentials induced and the depolarization during $100 \mathrm{~Hz}$ tetanus were not significantly different between the two areas (supplemental Fig. 4, available at www. jneurosci.org as supplemental material).

To determine whether voltage- and/or calcium-regulated potassium channels are different in CA2 neurons, we looked at several measures of their function. Spike accommodation is a defining feature of regular spiking pyramidal neurons (Cole and Nicoll, 1983) and is thought to require calcium-gated potassium channels (Lancaster et al., 1991). This measure, expressed as a ratio of the first interval between action potentials, was not different between CA1 and CA2 neurons (Fig. $5 C, D$ ). Action potential widths (CA1, $3.81 \pm 0.09 \mathrm{~ms}$; CA2, $3.96 \pm 0.08 \mathrm{~ms})$, which rely more significantly on voltage-gated potassium channels (Adams et al., 1982; Storm, 1987), and amplitudes (CA1, $104.86 \pm$ $3.92 \mathrm{mV}$; CA2, $100.89 \pm 3.6 \mathrm{mV} ; n=20$ each) did not differ significantly between CA1 and CA2 either. These data show that, when assessed for measures of potassium channels not included in the two-pore domain family, CA2 neurons are very similar to CA1 neurons.

Because whole-cell patch-clamping neurons necessarily disrupts intracellular components, and the dendrites of CA2 neurons are difficult to precisely localize for field potential recordings, we sought a measure of activity-dependent kinase activity that was free from possible in vitro artifact and independent of electrical stimulation. To do this, we stained brain tissue from animals that had experienced induced seizures using antibodies specific for the dually phosphorylated, active form of ERK. Although such experiments would not specifically address LTP-dependent pathways (Dudek and Fields, 2001; Thiels et al., 2002), the findings could support our assertion that the cellular and molecular properties of CA2 neurons, which include high levels of TREK-1, calbindin, adenosine receptors, and STEP, influence cell signaling in a way consistent with the lack of LTP and/or LTD in some neurons. The result could further shed some light on whether the resistance of CA2 to damage from a variety of insults potentially begins immediately on the onset of the insult, or whether distinct prolonged prosurvival programs are required to confer resistance to insults. We found that, despite robust staining for the phosphorylated ERK in CA1 and CA3 15 min after seizure activity, very few neurons in CA2 neurons were similarly labeled (supplemental Fig. 5, available at www.jneurosci.org as supplemental 

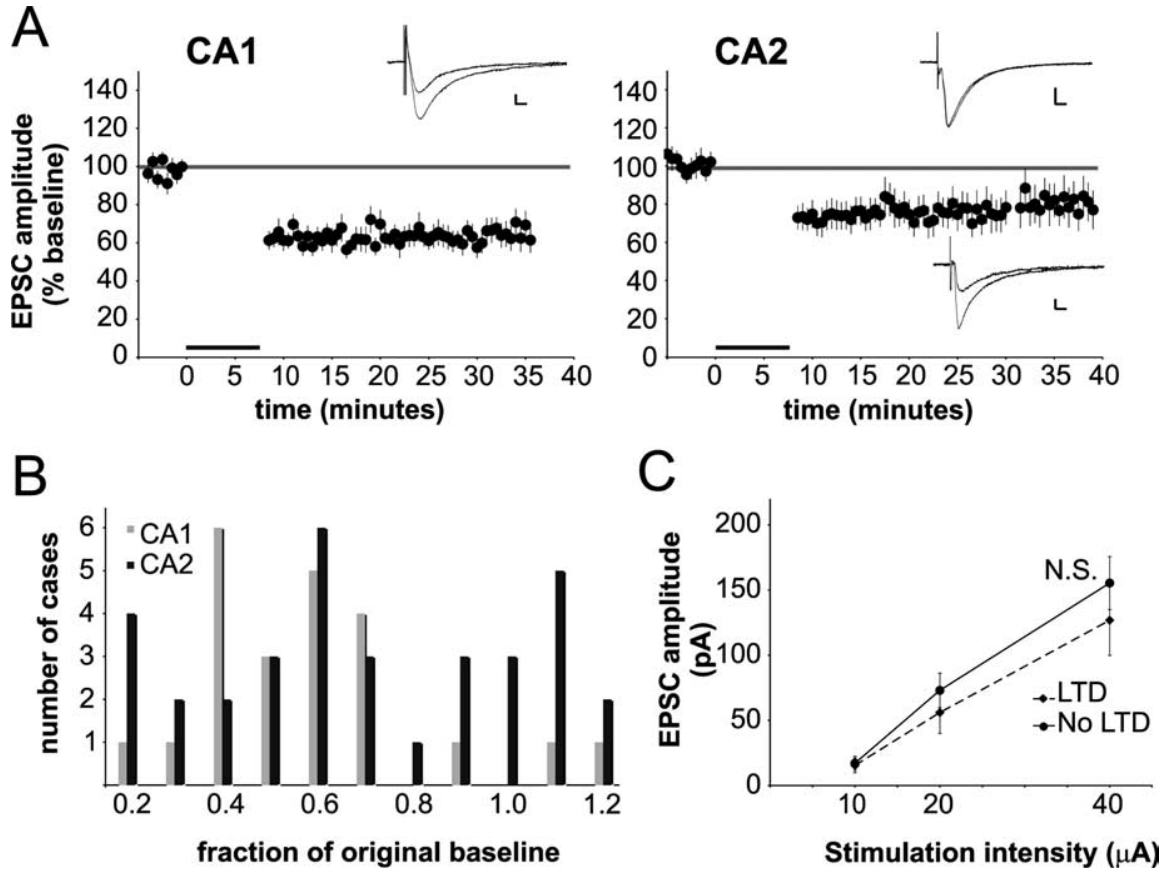

Figure 3. Long-term depression is impaired in some CA2 neurons. Shown compared with LTD in CA1 neurons, LTD was induced in less than two-thirds of CA2 neurons. $\boldsymbol{A}$, Average change in synaptic response to $2 \mathrm{~Hz}$ stimulation in cells clamped at $-75 \mathrm{mV}$. Data are from 23 CA1 neurons and 34 CA2 neurons. Representative traces from cases in which LTD was induced (CA1 and CA2) and not induced (CA2), before, and 20 min after LTD-inducing stimulation are shown as insets. Calibration: 10 pA (CA1 and CA2 LTD) and $25 \mathrm{pA}$ (CA2, no LTD), $10 \mathrm{~ms}$. Portions of the stimulus artifacts were removed for clarity of the figure. $\boldsymbol{B}$, Number of cases depressing after low-frequency stimulation. The distribution of cases in CA2 falls into a bimodal distribution with 13 of 34 cases failing to change significantly from baseline (black). Data from CA1 are shown for comparison (gray). The difference in the proportion of neurons expressing LTD between CA1 and CA2 is significant (Fisher's exact, $p=0.039$; unequal variance $t$ test, $p<$ 0.05). C, Post hoc analysis of initial EPSC sizes at three different intensities in neurons in which LTD was induced (LTD; dashed line) or not induced (no-LTD; solid line) ( $n=6$ in each case). None are significantly different ( $p>0.1$ at all stimulation intensities). Error bars indicate SEM.

material). These findings show that differences between CA2 neurons and their neighboring cells are apparent immediately after a pathological event and support the notion that the CA2 neurons fundamentally differ from CA1 and CA3 neurons in cell excitability, calcium buffering, and/or phosphatase activity. Primarily, however, they illustrate that many of the fundamental differences between CA1 and CA2 are not attributable to artifacts related to cutting the tissue and maintaining it in vitro.

\section{Discussion}

Without a doubt, the most striking characteristic of CA2 pyramidal neurons was the relative stability of their synaptic responses when compared with CA1 and CA3. This includes a severe deficit in LTP induction or expression and a heterogeneous response to LTD-inducing stimulation, neither of which could have been predicted based on the anatomic differences between CA2 neurons and those in CA3 and CA1, although in hindsight, the results are quite consistent with the complement of some molecules in CA2. Although we found that more current was required for action potential generation in CA2 cells, and this would have been an obvious reason for why LTP induction is impaired, two lines of evidence argue against the idea: (1) a pairing protocol, which involves voltage clamping the neuron to $0 \mathrm{mV}$ during stimulation with QX314 to block sodium-dependent action potentials, is equally ineffective at inducing LTP as are attempts using a $100 \mathrm{~Hz}$ protocol in current clamp, and (2) the number of action potentials and depolarization induced with $100 \mathrm{~Hz}$ stim- ulation recorded in CA2 were not different from those evoked in CA1. Thus, if TREK-1 or other two-pore domain channel-mediated current leak is the factor limiting LTP and/or LTD induction in CA2, it is likely to be doing so in the dendrites or by regulating other voltagedependent channels and not by influencing somatic action potentials. The total number of action potentials could, however, impact ERK activation in that the two are intimately linked (Zhao et al., 2005). Active and passive dendritic currents also remain a possible site of plasticity regulation in CA2, but additional work using recordings from dendrites will be required to rule out or support this hypothesis. In somatic whole-cell recordings, errors relating to space clamp can be a problem, and our recordings from these neurons with large dendrites are certainly not exempt (Spruston et al., 1993). For several reasons, however, we do not think these problems change the major finding of our study, that plasticity is very different in CA2. First, the degree of branching in CA1 and CA2 neurons is similar on a gross structural level (Ishizuka et al., 1995), and so the two areas can be considered comparable at least to some degree. Furthermore, we did perform one of our LTP protocols (the LTP-pairing protocol) with cesium in our internal solution, which would decrease a portion of the voltage-clamp error; we found that it made no difference in our ability to induce LTP. Errors in voltage- and space-clamp aside, synaptic responses can be compared in the same neuron before and after a "plasticity-inducing" stimulation. Nevertheless, our greatest concern is related to our finding that CA2 neurons are "leakier" and thus neurons in CA1 and CA2 may not be directly comparable in their recording errors. Because the mEPSCs from the very distal synapses may fall below our detection because of dampening of the signal by dendritic cable properties and leak potassium channels, we may be underestimating the number and the amplitudes of mEPSCs in CA2 (or overestimating the amplitudes, if small amplitude mEPSCs were not detected and therefore not included in the analysis). Again, however, we do not think these issues are likely to change our main conclusion, that plasticity is fundamentally different in CA2 from other CA regions. By using both evoked and miniature EPSCs to measure presynaptic and postsynaptic function, including NMDA receptor components, we may conclude that gross synaptic function cannot readily explain the difference in plasticity. We also note that, although our lack of LTP in CA2 was observed at near-physiological temperatures, most of our recordings were made at room temperature, thus potentially impacting some results of our study. We find the possibility of an impact of temperature that is differentially expressed in CA2 unlikely, however.

The impairment of LTP stabilization at later time points in mouse CA2, and the lack of long-term synaptic potentiation entirely in rat CA2, has many possible molecular causes: (1) $A_{1}$ 
A

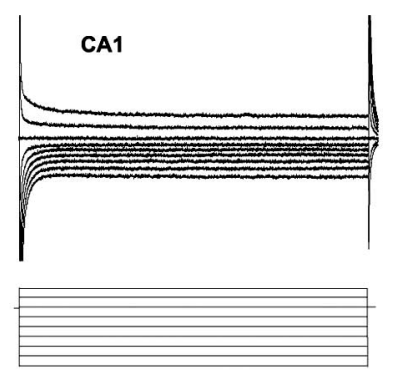

B

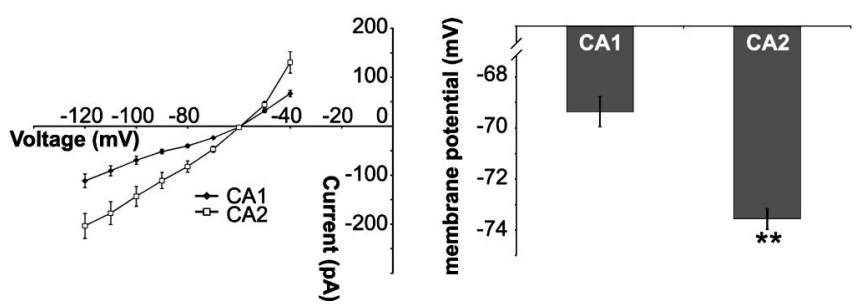

Figure 4. Passive membrane properties of $\mathrm{CA} 2$ neurons are different from those in CA1. $\boldsymbol{A}_{\text {, }}$ Leak currents were recorded from CA1 and $C A 2$ neurons with sodium gluconate substituted for sodium chloride, $\mathrm{CoCl}_{2}$, and ZD7288 to isolate leak potassium conductances. Currents were in response to 1-s-long voltage steps from -120 to $-40 \mathrm{mV}$ in $10 \mathrm{mV}$ increments. Representative traces are shown. Calibration: $50 \mathrm{~ms}, 100 \mathrm{pA}$. Steady-state levels were plotted in $\boldsymbol{B} . n=10$ each for CA1 and CA2. C, Resting membrane potentials were significantly more negative in CA2 neurons than in CA1 neurons. ${ }^{* *} p<0.001 ; n=20$ cells each. CA3 neurons had intermediate leak currents and membrane potentials $(n=10)$ (data not shown). Error bars indicate SEM.

adenosine receptors, which inhibit LTP consolidation (Arai et al., 1990; de Mendonca and Ribeiro, 1990) and/or synaptic transmission in general (Mitchell et al., 1993) are abundant in CA2 (Ochiishi et al., 1999); (2) extracellular matrix proteins, thought to play a role in restricting plasticity (Pizzorusso et al., 2002), are high in CA2 (Yamamoto et al., 1988; Bruckner et al., 2003); (3) oligodendrocytes (Berger and Frotscher, 1994) and astrocytes (Yamamoto et al., 1988) are distinct in CA2; (4) calbindin, a calcium binding protein more commonly found in inhibitory interneurons, is present in CA2 pyramidal cells (Leranth and Ribak, 1991; Toth and Freund, 1992) and can regulate synaptic plasticity (Chard et al., 1995); (5) STEP, a phosphatase that can dephosphorylate and inactivate ERK in response to NMDA receptor activation (Paul et al., 2003) and prevents LTP induction by inhibiting NMDA receptor function (Pelkey et al., 2002), is enriched in CA2 (Fig. 1) and (Boulanger et al., 1995); and (6) CA2 neurons have a different complement of integrin subunits than those in CA 3 and CA1, particularly in their lack of the $\beta 5$ integrin subunit (Pinkstaff et al., 1999). Integrin molecules may be critical for maintenance of LTP-related changes in the actin cytoskeleton (Kramar et al., 2006), and the decay of potentiation in mouse CA2 over $10 \mathrm{~min}$ is certainly consistent with the idea that CA2 is missing critical LTP-stabilizing components like integrins. Future experiments will be necessary to determine whether one or several of the molecules in the "conspiracy against plasticity" in CA2 contributes to the deficit in LTP and the low levels of ERK phosphorylation after seizure activity.

Although LTP was absent in nearly all CA2 neurons, the incidence of LTD at CA2 synapses fell into a heterogeneous distribution: those neurons expressing LTD, and those that did not. Such heterogeneity of this type of plasticity, and that neurons in CA2 express chromogranin-A and vasopressin $1 \mathrm{~b}$

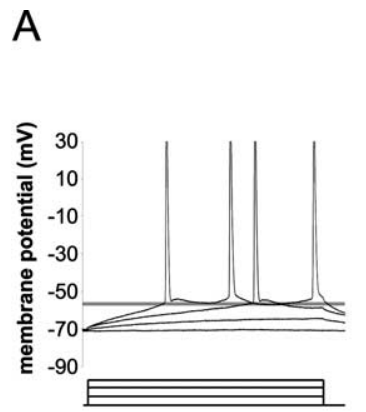

B
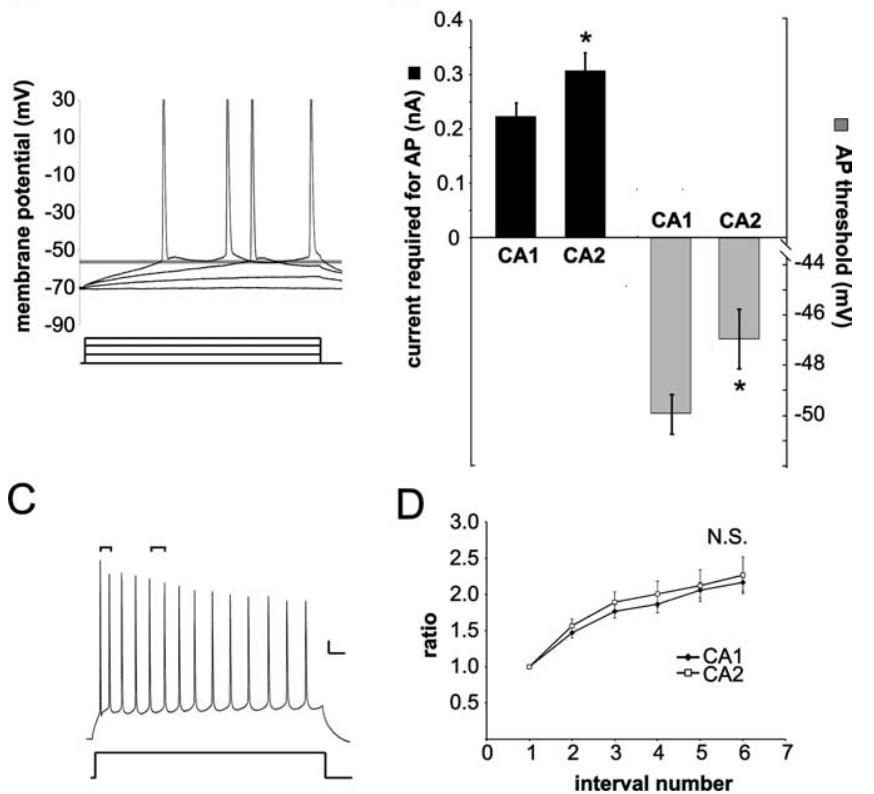

Figure 5. Action potential properties in CA1 and CA2 neurons. Action potentials, recorded in current-clamp mode, were induced with 180 -ms-long current pulses in $0.2 \mathrm{nA}$ increments. An example is shown in $\boldsymbol{A}$. $\boldsymbol{B}$, The minimum current required to generate action potentials, and the membrane potential threshold that must be reached are compared in CA1 and CA2 neurons $\left({ }^{*} p<0.05 ; n=20\right.$ in both cases). D, Spike accommodation, measured as a ratio of the interval between the first two spikes induced with a 700-ms-long depolarization, was not significantly different between CA1 and CA2. An example is shown in C. Calibration: $50 \mathrm{~ms}, 10 \mathrm{mV}$. Error bars indicate SEM.

receptors in a similar heterogeneous manner, indicate that the pyramidal neurons within CA2 are indeed a mixed population of cells (Munoz, 1990; Young et al., 2006). That LTD can be induced in some neurons and not others also suggests that the extracellular matrix and glial components, on a gross level, still support some plasticity in CA2 stratum radiatum. Our own staining for pERK reveals a small number of neurons in CA2 that stain after seizures, but at this time we are unable to predict whether this population represents one of the LTD phenotypes. Whether and how LTD could be reversed without LTP should be a topic of additional study, but investigations on this effect are generally hampered by the fact that LTP can "wash out" by typical patch recording longer than a few minutes, and field potential recording would not distinguish among the different populations of CA2 neurons.

Synaptic plasticity such as LTP and LTD and ERK phosphorylation require rises in intracellular calcium postsynaptically, most commonly through NMDARs, and so unique expression, processing, or regulation of NMDARs may be one way that plasticity is reduced in CA2. This explanation is particularly attractive given that STEP, which is found concentrated in CA2 (Boulanger et al., 1995), blocks LTP induction by reducing NMDAR currents (Pelkey et al., 2002). We found that this explanation was unlikely to be the sole cause of the synaptic stability of CA2, however; NMDAR-mediated currents were similar to those recorded from CA1 neurons and thus cannot explain the difference in synaptic plasticity in the two areas. The role of STEP in dephosphorylation and inactivation of ERK (Paul et al., 2003), although a likely explanation for the reduced staining for pERK in CA2 after seizures, may not be what is directly interfering with LTP; okadaic acid, 
which blocks the phosphatase upstream of STEP, did not aid in LTP induction in CA2. Lack of large differences between CA1 and CA2 in non-NMDAR-dependent responses and presynaptic function, as assessed by paired-pulse facilitation and mEPSC frequency, are likewise unable to explain a lack of plasticity. This leads us to expect that any one or a combination of the several genes differentially expressed in CA2 neurons postsynaptically could be critical factors in both plasticity and resistance to neuronal injury (Lein et al., 2004, 2005). Future study of CA2 may therefore reveal much about the cellular processes of synaptic plasticity and cell death (Kirino, 1982; Sadowski et al., 1999; Maxwell et al., 2003), but in addition, it may also reveal new and previously unpredicted insights into hippocampal function.

\section{References}

Adams PR, Constanti A, Brown DA, Clark RB (1982) Intracellular $\mathrm{Ca}^{2+}$ activates a fast voltage-sensitive $\mathrm{K}^{+}$current in vertebrate sympathetic neurons. Nature 296:746-749.

Alger BE, Teyler TJ (1976) Long-term and short-term plasticity in the CA1, $\mathrm{CA} 3$, and dentate regions of the rat hippocampal slice. Brain Res 110:463-480.

Arai A, Kessler M, Lynch G (1990) The effects of adenosine on the development of long-term potentiation. Neurosci Lett 199:41-44.

Asztely F, Xiao MY, Gustafsson B (1996) Long-term potentiation and paired-pulse facilitation in the hippocampal CA1 region. NeuroReport 7:1609-1612.

Benes FM, Todtenkopf MS (1999) Effect of age and neuroleptics on tyrosine hydroxylase-IR in sector CA2 of schizophrenic brain. NeuroReport 10:3527-3530.

Benes FM, Kwok EW, Vincent SL, Todtenkopf MS (1998) A reduction of nonpyramidal cells in sector CA2 of schizophrenics and manic depressives. Biol Psychiatry 44:88-97.

Berger T, Frotscher M (1994) Distribution and morphological characteristics of oligodendrocytes in the rat hippocampus in situ and in vitro: an immunocytochemical study with the monoclonal Rip antibody. J Neurocytol 23:61-74

Bliss TV, Lomo T (1973) Long-lasting potentiation of synaptic transmission in the dentate area of the anaesthetized rabbit following stimulation of the perforant path. J Physiol (Lond) 232:331-356.

Boulanger LM, Lombroso PJ, Raghunathan A, During MJ, Wahle P, Naegele JR (1995) Cellular and molecular characterization of a brain-enriched protein tyrosine phosphatase. J Neurosci 15:1532-1544.

Braak H (1980) Architectonics of the human telencephalic cortex. Berlin: Springer.

Bruckner G, Grosche J, Hartlage-Rubsamen M, Schmidt S, Schachner M (2003) Region and lamina-specific distribution of extracellular matrix proteoglycans, hyaluronan and tenascin- $\mathrm{R}$ in the mouse hippocampal formation. J Chem Neuroanat 26:37-50.

Buller AL, Larson HC, Schneider BE, Beaton JA, Morrisett RA, Monaghan DT (1994) The molecular basis of NMDA receptor subtypes: native receptor diversity is predicted by subunit composition. J Neurosci 14:5471-5484.

Chard PS, Jordan J, Marcuccilli CJ, Miller RJ, Leiden JM, Roos RP, Ghadge GD (1995) Regulation of excitatory transmission at hippocampal synapses by calbindin D28k. Proc Natl Acad Sci USA 92:5144-5448.

Cole AE, Nicoll RA (1983) Acetylcholine mediates a slow synaptic potential in hippocampal pyramidal cells. Science 221:1299-1301.

de Mendonca A, Ribeiro JA (1990) 2-Chloroadenosine decreases long-term potentiation in the hippocampal CA1 area of the rat. Neurosci Lett 118:107-111.

Dudek SM, Fields RD (2001) Mitogen-activated protein kinase/extracellular signal-regulated kinase activation in somatodendritic compartments: roles of action potentials, frequency, and mode of calcium entry. J Neurosci 21:RC122(1-5)

Goldman DE (1943) Potential, impedance, and rectification in membranes. J Gen Phys 27:37-60.

Han C, Kasai N, Torimitsu K (2005) CA2: the most vulnerable sector to bicuculline exposure in rat hippocampal slice cultures. NeuroReport $16: 333-336$.
Henze DA, Gonzalez-Burgos GR, Urban NN, Lewis DA, Barrionuevo G (2000) Dopamine increases excitability of pyramidal neurons in primate prefrontal cortex. J Neurophysiol 84:2799-2809.

Ishizuka N, Cowan WM, Amaral DG (1995) A quantitative analysis of the dendritic organization of pyramidal cells in the rat hippocampus. J Comp Neurol 362:17-45.

Kirino T (1982) Delayed neuronal death in the gerbil hippocampus following ischemia. Brain Res 239:57-69.

Kramar EA, Lin B, Rex CS, Gall CM, Lynch G (2006) Integrin-driven actin polymerization consolidates long-term potentiation. Proc Natl Acad Sci USA 103:5579-5584.

Lancaster B, Nicoll RA, Perkel DJ (1991) Calcium activates two types of potassium channels in rat hippocampal neurons in culture. J Neurosci 11:23-30.

Lein ES, Zhao X, Gage FH (2004) Defining a molecular atlas of the hippocampus using DNA microarrays and high-throughput in situ hybridization. J Neurosci 24:3879-3889.

Lein ES, Callaway EM, Albright TD, Gage FH (2005) Redefining the boundaries of the hippocampal CA2 subfield in the mouse using gene expression and 3-dimensional reconstruction. J Comp Neurol 485:1-10.

Leranth C, Ribak CE (1991) Calcium-binding proteins are concentrated in the CA2 field of the monkey hippocampus: a possible key to this region's resistance to epileptic damage. Exp Brain Res 85:129-136.

Liu L, Wong TP, Pozza MF, Lingenhoehl K, Wang Y, Sheng M, Auberson YP, Wang YT (2004) Role of NMDA receptor subtypes in governing the direction of hippocampal synaptic plasticity. Science 304:1021-1024.

Lorente de No R (1934) Studies on the structure of the cerebral cortex II. Continuation of the study of the ammonic system. J Psychol Neurol (Leipzig) 46:113-117.

Malenka RC, Bear MF (2004) LTP and LTD: an embarrassment of riches. Neuron 44:5-21.

Maxwell WL, Dhillon K, Harper L, Espin J, MacIntosh TK, Smith DH, Graham DI (2003) There is differential loss of pyramidal cells from the human hippocampus with survival after blunt head injury. J Neuropathol Exp Neurol 62:272-279.

Mitchell JB, Miller K, Dunwiddie TV (1993) Adenosine-induced suppression of synaptic responses and the initiation and expression of long-term potentiation in the CAl region of the hippocampus. Hippocampus 3:77-86.

Munoz DG (1990) The distribution of chromogranin A-like immunoreactivity in the human hippocampus coincides with the pattern of resistance to epilepsy-induced neuronal damage. Ann Neurol $27: 266-275$.

Ochiishi T, Saitoh Y, Yukawa A, Saji M, Ren Y, Shirao T, Miyamoto H, Nakata H, Sekino Y (1999) High level of adenosine Al receptor-like immunoreactivity in the CA2/CA3a region of the adult rat hippocampus. Neuroscience 93:955-967.

Paul S, Nairn AC, Wang P, Lombroso PJ (2003) NMDA-mediated activation of the tyrosine phosphatase STEP regulates the duration of ERK signaling. Nat Neurosci 6:34-42.

Pelkey KA, Askalan R, Paul S, Kalia LV, Nguyen TH, Pitcher GM, Salter MW, Lombroso PJ (2002) Tyrosine phosphatase STEP is a tonic brake on induction of long-term potentiation. Neuron 34:127-138.

Pinkstaff JK, Detterich J, Lynch G, Gall C (1999) Integrin subunit gene expression is regionally differentiated in adult brain. J Neurosci 19:1541-1556.

Pizzorusso T, Medini P, Berardi N, Chierzi S, Fawcett JW, Maffei L (2002) Reactivation of ocular dominance plasticity in the adult visual cortex. Science 298:1248-1251.

Racine R (1972) Modification of seizure activity by electrical stimulation. II. Motor seizure. Electroencephalogr Clin Neurophysiol 32:281-294.

Sadowski M, Wisniewski HM, Jakubowska-Sadowska K, Tarnawski M, Lazarewicz JW, Mossakowski MJ (1999) Pattern of neuronal loss in the rat hippocampus following experimental cardiac arrest-induced ischemia. J Neurolog Sci 168:13-20.

Schulz PE, Cook EP, Johnston D (1994) Changes in paired-pulse facilitation suggest presynaptic involvement in long-term potentiation. J Neurosci 14:5325-5337.

Spruston N, Jaffe DB, Williams SH, Johnston D (1993) Voltage- and spaceclamp errors associated with the measurement of electrotonically remote synaptic events. J Neurophysiol 70:781-802. 
Storm JF (1987) Action potential repolarization and a fast afterhyperpolarization in rat hippocampal pyramidal cells. J Physiol (Lond) 385:733-759.

Talley EM, Solorzano G, Lei Q, Kim D, Bayliss DA (2001) CNS distribution of members of the two-pore-domain (KCNK) potassium channel family. J Neurosci 21:7491-7505.

Thiels E, Kanterewicz BI, Norman ED, Trzaskos JM, Klann E (2002) Longterm depression in the adult hippocampus in vivo involves activation of extracellular signal-regulated kinase and phosphorylation of Elk-1. J Neurosci 22:2054-2062.

Toth K, Freund TF (1992) Calbindin D28k-containing nonpyramidal cells in the rat hippocampus: their immunoreactivity for GABA and projection to the medial septum. Neuroscience 49:793-805.

Wenzel A, Scheurer L, Kunzi R, Fritschy JM, Mohler H, Benke D (1995) Distribution of NMDA receptor subunit proteins NR2A, 2B, 2C and 2D in rat brain. NeuroReport 7:45-48.
Woodhams PL, Celio MR, Ulfig N, Witter MP (1993) Morphological and functional correlates of borders in the entorhinal cortex and hippocampus. Hippocampus 3:303-312.

Yamamoto M, Marshall P, Hemmendinger LM, Boyer AB, Caviness VSJ (1988) Distribution of glucuronic acid-and sulfate-containing glycoproteins in the central nervous system of the adult mouse. Neurosci Res 5:273-298.

Young WS, Li J, Wersinger SR, Palkovits M (2006) The vasopressin $1 \mathrm{~b}$ receptor is prominent in the hippocampal area CA2 where it is unaffected by restraint stress or adrenalectomy. Neuroscience 143:1031-1039.

Zhao M, Adams JP, Dudek SM (2005) Pattern-dependent role of NMDA receptors in action potential generation: consequences on extracellular signal-regulated kinase activation. J Neurosci 25:7032-7039.

Zhao X, Lein ES, He A, Smith SC, Aston C, Gage FH (2001) Transcriptional profiling reveals strict boundaries between hippocampal subregions. J Comp Neurol 441:187-196. 